\title{
Development, construction and qualification tests of the mechanical structures of the electromagnetic calorimeter of the Mu2e experiment at Fermilab
}

\author{
N. Atanov ${ }^{1}$, V. Baranov ${ }^{1}$, L. Borrel ${ }^{2}$, C. Bloise ${ }^{3}$, J. Budagov $^{1}$, S. Ceravolo ${ }^{3}$, F. \\ Cervelli $^{4}$, F. Colao ${ }^{5}$, M. Cordelli ${ }^{3}$, G. Corradi ${ }^{3}$, Y. I. Davydov ${ }^{1}$, S. Di Falco ${ }^{4}$, E. \\ Diociaiuti $^{3}$, S. Donati ${ }^{4}, 6$, B. Echenard ${ }^{2}$, C. Ferrari ${ }^{4}$, A. Gioiosa ${ }^{4}$, S. Giovannella ${ }^{3}$, V. \\ Giusti $^{4}$, V. Glagolev ${ }^{1}$, F. Grancagnolo ${ }^{7}$, D. Hampai ${ }^{3}$, F. Happacher ${ }^{3}$, D. Hitlin ${ }^{2}$, D. Lin ${ }^{2}$, \\ M. Martini ${ }^{3,8}$, S. Middleton ${ }^{2}$, S. Miscetti ${ }^{3}$, L. Morescalchi ${ }^{4}$, D. Paesani ${ }^{3}$, D. \\ Pasciuto $^{4,6, *}$, E. Pedreschi ${ }^{4}$, F. Porter ${ }^{2}$, F. Raffaelli ${ }^{4}$, A. Saputi ${ }^{3}$, I. Sarra ${ }^{3}$, F. \\ Spinella $^{4}$,A. Taffara ${ }^{4}$, G. F. Tassielli ${ }^{7}$, V. Tereshchenko ${ }^{1}$, Z. Usubov ${ }^{1}$, I. I. Vasilyev ${ }^{1}$, A. \\ Zanetti $^{9}$, R. Y. Zhu ${ }^{2}$ \\ on behalf of the Mu2e Collaboration \\ ${ }^{1}$ Joint Institute for Nuclear Research, Dubna, Russia \\ ${ }^{2}$ California Institute of Technology, Pasadena, California, United States \\ ${ }^{3}$ Laboratori Nazionali di Frascati dell'INFN, Frascati, Italy \\ ${ }^{4}$ INFN - Sezione di Pisa, Pisa, Italy \\ ${ }^{5}$ ENEA - Frascati, Frascati, Italy \\ ${ }^{6}$ Department of Physics, University of Pisa, Pisa, Italy \\ ${ }^{7}$ INFN - Sezione di Lecce, Lecce, Italy \\ ${ }^{8}$ Department of Engineering Sciences, Guglielmo Marconi University, Rome, Italy \\ ${ }^{9}$ INFN - Sezione di Trieste, Trieste, Italy \\ E-mail: daniele.pasciuto@pi.infn.it
}

The "muon-to-electron conversion" (Mu2e) experiment at Fermilab will search for the Charged Lepton Flavour Violating neutrino-less coherent conversion of a muon into an electron in the field of an aluminum nucleus. The electromagnetic calorimeter is one of the three Mu2e detectors. The hostile Mu2e operational conditions, in terms of radiation levels (total ionizing dose of 12 $\mathrm{krad}$ and a neutron fluence of $\left.5 \times 10^{10} \mathrm{n} / \mathrm{cm}^{2} @ 1 \mathrm{MeVeq}(\mathrm{Si}) / \mathrm{y}\right)$, magnetic field intensity $(1 \mathrm{~T})$ and vacuum level $\left(10^{-4}\right.$ Torr) have posed tight constraints on the design of the detector mechanical structures and materials choice. In this report the mechanical overall description of the calorimeter is presented, such as the qualification tests performed during the construction of its components and the realized technological choices.

\footnotetext{
*** Particles and Nuclei International Conference - PANIC2021 ***

*** 5 - 10 September, $2021 * * *$

*** Online ***
}

\footnotetext{
*Corresponding author.
} 


\section{The Mu2e experiment and the Calorimeter}

The "muon-to-electron conversion" (Mu2e) experiment at Fermilab will search for the Charged Lepton Flavour Violating neutrino-less coherent conversion of a muon into an electron in the field of an aluminum nucleus. The observation of this process would be the unambiguous evidence of New Physics. Mu2e detectors comprise a straw-tracker, an electromagnetic calorimeter (Figure 1) [1] located inside a vessel with a $10^{-4}$ Torr vacuum level surrounded by a large superconducting solenoid which generates an axial magnetic field of $1 \mathrm{~T}$, and an external veto for cosmic rays. The main calorimeter function is providing complementary information to the tracker to achieve a
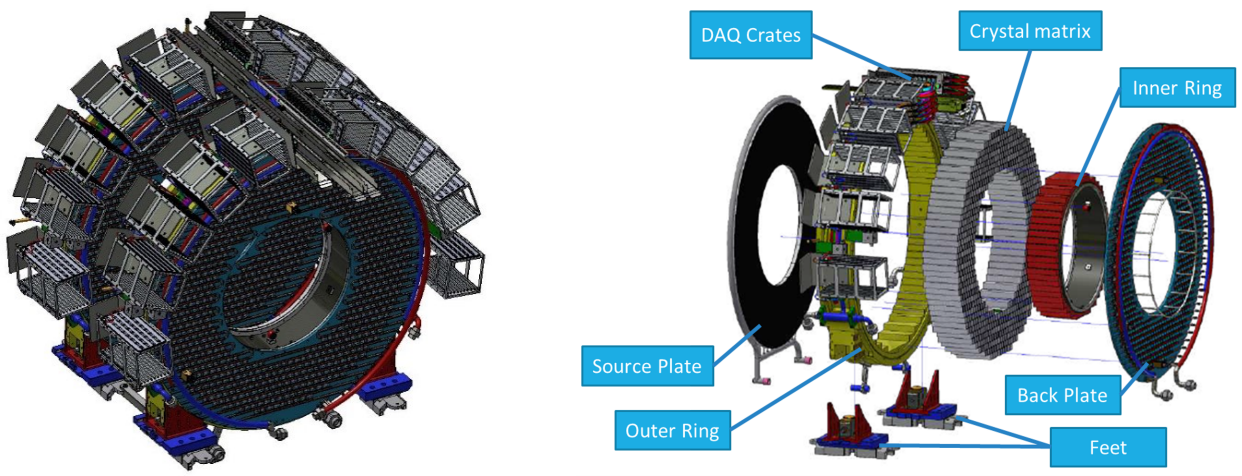

Figure 1: CAD drawings of the Mu2e electromagnetic calorimeter. Global view of the two disks (left). Exploded view of one disk: the Outer Ring, the Source Plate, the Crystal Matrix, the DAQ Crates, the Inner Ring, the Back Plate and the Feet are shown (right).

powerful $\mu /$ e separation which is crucial to extract the conversion electron signal from the expected overwhelming background. The calorimeter is also exploited in a calorimeter-seeded track finder algorithm which improves track reconstruction efficiency and makes the algorithm more robust in high detector occupancy conditions. Moreover, the calorimeter is used to implement a fast online standalone trigger independent from the tracker. These tasks translate into the following requirements for $105 \mathrm{MeV}$ electrons: (a) large geometric acceptance; (b) time resolution better than $500 \mathrm{ps}$; (c) energy resolution better than $10 \%$ and (d) position resolution of the order of $1 \mathrm{~cm}$.

The detector has been designed as a state-of-the-art crystal calorimeter and employs 1348 pure Cesium Iodide (CsI) crystals readout by UV-extended silicon photosensors (SiPM) and fast frontend and digitization electronics. A design consisting of two identical annular matrices (named "disks") positioned at the relative distance of $70 \mathrm{~cm}$ downstream the aluminum target along the muon beamline satisfies the Mu2e physics requirements.

The hostile Mu2e operational conditions, in terms of radiation levels (total expected ionizing dose of $12 \mathrm{krad}$ and a neutron fluence of $\left.5 \times 10^{10} \mathrm{n} / \mathrm{cm}^{2} @ 1 \mathrm{MeVeq}(\mathrm{Si}) / \mathrm{y}\right)$, magnetic field intensity (1 $\mathrm{T})$ and vacuum level $\left(10^{-4}\right.$ Torr $)$ have posed tight constraints on scintillating materials, sensors, electronics and on the design of the detector mechanical structures and material choice.

\section{Calorimeter components and performed tests}

The support structure of each 674 crystal matrix is composed of an aluminum hollow ring and parts made of open-cell vacuum-compatible carbon fiber. The photosensors and front-end 
electronics for the readout of each crystal are inserted in a machined copper holder and make a unique mechanical unit. The resulting 674 mechanical units are supported by a machined plate of vacuum-compatible plastic material. The plate also integrates the cooling system made of a network of copper lines flowing a low temperature radiation-hard fluid and placed in thermal contact with the copper holders to constitute a low resistance thermal bridge. The data acquisition electronics are hosted in aluminum custom crates positioned on the external lateral surface of the disks. The crates also integrate the electronics cooling system as lines running in parallel to the front-end system.

The heart of each of the disks is the ring-shaped matrix of 674 un-doped CsI crystals $(34 \times 34 \times 200$ $\mathrm{mm}^{3}$ ) which has an internal/external diameter of $650 \mathrm{~mm} / 1314 \mathrm{~mm}$. Visual and dimensional inspection were executed on each crystal, such as the measurements of the optical properties and Radiation Induced Noise (RIN) [2]. Crystals are wrapped in Tyvek foils (150 $\mu \mathrm{m}$ thick) to improve internal light reflection and separated vertically and horizontally with black Tedlar foils ( $50 \mu \mathrm{m}$ thick) to minimize optical cross-talk. Tests for crystal piling up and manipulation have been performed [3]. The crystal matrix is externally supported by the Outer Ring made of Al6082 aluminum. The outer diameter and the thickness of the ring are $1460 \mathrm{~mm}$ and $146 \mathrm{~mm}$, respectively. The Outer Ring also provides all the fastening features for the other components. It hosts the manifolds for the crate cooling system and supports the DAQ crates on the lateral surface.

The material choice and budget of the mechanical structures that can be traversed by the particles have been optimized to minimize particle energy losses. The two components placed on the particle trajectories are the Source Panel, which is the frontal cover of the each crystal matrix, and the Inner Ring, which occupies the inner bore surface. They are both made of carbon fiber planes strengthened by light aluminum structures when necessary.

The Source Plate supports 10 thin-wall (0.375" OD x 0.02"thickness) aluminum tubes symmetrically arranged on each disk to flow the calibration source fluid (CF-770). It also provides the frontal enclosure for crystal protection. The Source Plate is made of a sandwich with $1.4 \mathrm{~mm}$ carbon fiber skins and a core of aluminum honeycomb (series 3003) $22 \mathrm{~mm}$ thick, 3/8" cell size, and 0.003" wall thickness. The expected energy loss is $1.2 \mathrm{MeV}$ for $100 \mathrm{MeV}$ electrons.

The Inner Ring performs a fundamental function for the support and alignment of the crystal matrix. It is made of a carbon fiber cylindrical skin with an internal diameter of $712 \mathrm{~mm}, 4.2 \mathrm{~mm}$ thick, an F-.220/193/50 CF fabric (0/90 ${ }^{\circ}$ texture) with cyanate ester resin; two $5083 \mathrm{H} 111$ aluminum alloy reinforcement internal rings with an internal diameter of $672 \mathrm{~mm}$, an outer diameter of $712 \mathrm{~mm}, 13$ $\mathrm{mm}$ thick to increase its stiffness; three outer step ribs made of a sandwich slab with $1.4 \mathrm{~mm}$ carbon fiber skins (same as the cylindrical skin) and a core of aluminum honeycomb (series 3003) $22 \mathrm{~mm}$ thick, 3/8" cell size, and 0.003" wall thickness. The Inner Ring is connected and supported by the Back Plate and Source Plate and provides the internal vertical/horizontal reference for the crystal matrix.

The Back Plate is the rear mechanical enclosure of the calorimeter. It also supports the 674 frontend units which include the SiPMs and front-end electronic boards. Each SiPM has been qualified with a dedicated set up both for geometrical and electrical properties [4]. The SiPM and front-end electronic modules are composed of 2 SiPMs glued on a copper holder, 2 front-end boards and a copper protective cage. The modules are fastened directly on the Back Plate Cooling lines to optimize thermal conductivity. The Back Plate is made of a milled PEEK plate (20 mm thick). PEEK was chosen to optimize thermal isolation of the electronics and for its good outgassing 
characteristics. The Back Plate integrates the cooling system of the front-end units. It embeds a network of vacuum brazed copper lines flowing fluid (3M Novec 649$)$ at $-15^{\circ} \mathrm{C}$ to minimize SiPM dark current and maintain an acceptable signal/noise ratio over the three years of data taking. Two AISI 316L I/O manifolds placed on the external border distribute the cooling fluid among the network of 38 parallel copper cooling lines embedded in the PEEK to maximize the temperature uniformity.

Each calorimeter disk supports 10 DAQ crates placed on its lateral surface to host 80 DAQ boards which digitize and transmit the data received from the front-end through optical fibers out of the vessel to the central DAQ system. Each crate integrates a network of cooling lines to remove the $320 \mathrm{~W}$ dissipated by the set of 8 DAQ boards. To reduce envelopes and optimize the system performance, the cooling lines are directly carved in the crate sides. Optimal thermal contact between the electronic components and the heat sink is achieved through a machined copper plate positioned on top of the DAQ boards and placed in thermal contact with the components with vacuum proof thermal grease (Apiezon). The DAQ crate structure is completed by a set of tungsten plates which protect the electronic components from the radiation present in the experimental area at run time. Thermal simulations and experimental tests have been performed to crosscheck the cooling system performance.

\section{Conclusions}

The design of the calorimeter is completed; most of the parts have already been built and tested, and some of them are already at Fermilab. Very few components are missing, but they are under construction. Calorimeter assembly will take place in a dedicated cleanroom at SiDet, a Fermilab laboratory, in spring 2022.

\section{Acknowledgments}

We are grateful for the vital contributions of the Fermilab staff and the technical staff of the participating institutions. This work was supported by the US D.O.E.; the I.N.F.N., Italy; the Science and Technology Facilities Council, UK; the Ministry of Education and Science, Russian Federation; the National Science Foundation, USA; the Thousand Talents Plan, China; the Helmholtz Association, Germany; and the EU Horizon 2020 Research and Innovation Program under the Marie Sklodowska-Curie Grant Agreement No. 690385 ,734303, 822185, 858199, 101003460. This document was prepared by members of the Mu2e Collaboration using the resources of the Fermi National Accelerator Laboratory (Fermilab), a U.S. D.O.E., Office of Science, HEP User Facility. Fermilab is managed by F.R.A., LLC (FRA), acting under Contract No. DE-AC02-07CH11359.

\section{References}

[1] L. Morescalchi et al., Final Design and Current..., PoS ICHEP2020 (2021) 759.

[2] N. Atanov et al., Quality assurance..., IEEE TNS 65.2 (2017): 752-757.

[3] D. Pasciuto, Physical and mechanical ..., PhD Thesis, Università Guglielmo Marconi (2021). 
[4] L. Morescalchi et al., Automated Test ..., PoS TWEPP2018 (2019) 017 10.22323/1.343.0017. 